\title{
Multilinguales
}

\section{Du petit poucet devenu l'enfant-océan a Mekideche et Baïdro effets d'intertextualité et de transculturalité}

From Tom-Thumb to the Ocean-Child in Mekideche and Baïdro: Intertextuality and Crosscultural Effects

\section{Marie-Agnès Thirard}

\section{(2) OpenEdition Journals}

Édition électronique

URL : http://journals.openedition.org/multilinguales/2579

DOI : $10.4000 /$ multilinguales. 2579

ISSN : 2335-1853

\section{Éditeur}

Université Abderrahmane Mira - Bejaia

\section{Édition imprimée}

Date de publication : 1 décembre 2013

Pagination : 123-137

ISSN : 2335-1535

Référence électronique

Marie-Agnès Thirard, « Du petit poucet devenu l'enfant-océan a Mekideche et Baïdro effets

d'intertextualité et de transculturalité », Multilinguales [En ligne], 2 | 2013, mis en ligne le 01 décembre 2013, consulté le 17 septembre 2019. URL : http://journals.openedition.org/multilinguales/2579 ; DOI : 10.4000/multilinguales.2579

Ce document a été généré automatiquement le 17 septembre 2019.

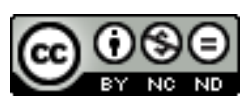

Multilinguales est mise à disposition selon les termes de la Licence Creative Commons Attribution Pas d'Utilisation Commerciale - Pas de Modification 4.0 International 


\title{
Du petit poucet devenu l'enfant-océan a Mekideche et Baïdro effets d'intertextualité et de transculturalité
}

\author{
From Tom-Thumb to the Ocean-Child in Mekideche and Baïdro: Intertextuality \\ and Crosscultural Effects
}

Marie-Agnès Thirard

1 Le Petit Poucet est sans aucun doute le plus doué des héros. Doté comme chacun sait d'une paire de bottes de sept lieues, il a donc allègrement vaincu les contraintes du temps et de l'espace, au point de franchir les montagnes des réécritures et d'évoluer aussi d'une rive à l'autre de la Méditerranée. Il parcourt depuis la nuit des temps, dans la tradition orale et dans la culture savante et populaire, l'univers des laissés pour compte et lutte sans vergogne contre des forces douées de bêtise et de méchanceté : sous le nom de Mékidèche, il parvient ainsi à vaincre l'ignoble ghoule en Afrique du Nord, voire l'ogre ou le diable en Occident. Cependant dans la forêt inextricable des contes, il sème au fil du temps quelques petits cailloux qui pourraient nous guider dans ce parcours au gré des effets d'intertextualité et d'interculturalité, voire de transculturalité.

\section{Le texte fondateur}

2 Le conte de Perrault, publié en 1697 dans le recueil des Histoires ou Contes du temps passé avec des moralités, connaît encore trois siècles plus tard un véritable succès et représente un hypotexte incontournable. Or si Perrault est bien le père littéraire de cet enfant surdoué mais doté de frères et sœurs innombrables dans la patrie des contes, on oublie trop souvent qu'une fée-marraine, alias Madame d'Aulnoy réécrivit à la même époque les aventures de Finette Cendron, sorte de Petit Poucet en jupons. Le temps 
n'est plus en effet où le seul nom du célèbre académicien suffisait à rendre compte de la mode des contes de fées à la fin du XVII ${ }^{\text {ème }}$ siècle. Si ces personnages étaient à l'origine destinés aux adultes cultivés et lettrés, ils furent ensuite récupérés dans la littérature de colportage avant de glisser dans le domaine de la littérature de jeunesse dès ses premiers balbutiements. C'est donc ces lointains ancêtres qui serviront de référents culturels et qui nous tiendront lieu de fil d'Ariane dans le labyrinthe narratif où quelques Minotaures sévissent encore!

3 Si dans la tradition populaire le thème des enfants abandonnés dans la forêt est très répandu, il n'en demeure pas moins que c'est la version de Charles Perrault qui est la plus connue en littérature et qu'elle a exercé une influence pour le moins prégnante sur les versions orales mais aussi sur les réécritures. Or le digne académicien a métamorphosé le personnage du petit faible mais rusé. Cette transmutation digne de l'alchimie de la plume s'opère dans le subtil creuset d'un humour féroce et d'un ancrage sociologique évident dans cette société de la fin du siècle de Louis XIV. Les fameuses bottes de sept lieues - qui finiront par symboliser le personnage et lui collent à la peau car, ne l'oublions jamais, elles étaient fées et avaient le don de «s'agrandir et de s'apetisser selon la jambe de celui qui les chaussait »; bottes sur mesure si l'on en croit notre conteur - sont non seulement le fruit d'une imagination délirante mais aussi le symbole d'une ascension sociale fulgurante. C'est que les bottes, à l'époque où le code vestimentaire est important, sont le privilège des nobles. En bottant les chats et en faisant de ce Petit Poucet une créature bottée à son tour, Perrault fait donc bien plus que lui donner un auxiliaire magique ; il lui permet une ascension sociale à l'image de celle de cette bourgeoisie montante à laquelle il appartient, laquelle vit dans l'orbite de la Cour. Il n'est donc point étonnant qu'une fois botté notre Petit Poucet non seulement s'enrichisse mais encore gravisse allègrement les marches de l'échelle sociale.

Chacun ici se souvient de cette double fin heureuse aux multiples possibilités narratives que le conteur nous propose avec beaucoup de distanciation et non sans quelque ironie. Dans un premier temps, le héros exerce un affreux chantage sur l'ogresse qui tient de l'extorsion de fonds : craignant de perdre son mari soi-disant attaqué par des voleurs qui exigent une rançon, «la bonne femme fort effrayée » donne tout ce qu'elle a «car cet ogre ne laissait pas d'être fort bon mari quoiqu'il mangeât les petits enfants » (Perrault, 1997 : p. 126).

5 Perrault propose cependant une autre fin plus révélatrice de cette société de fin de siècle :

(...) Lorsque le petit Poucet eut chaussé les bottes de l'ogre, il s'en alla à la Cour, où il savait qu'on était fort en peine d'une armée qui était à deux cents lieues de là, et du succès d'une bataille qu'on avait donnée. Il alla, disent-ils, trouver le roi, et lui dit que s'il le souhaitait, il lui rapporterait des nouvelles de l'armée avant la fin du jour. Le roi lui promit une grosse somme d'argent s'il en venait à bout. Le Petit Poucet rapporta des nouvelles dès le soir même, et cette première course l'ayant fait connaître, il gagnait tout ce qu'il voulait ; car le roi le payait parfaitement bien pour porter ses ordres à l'armée, et une infinité de dames lui donnait tout ce qu'il voulait pour avoir des nouvelles de leurs amants, et ce fut son plus grand gain. (Perrault, 1997 : p. 104)

6 Voilà donc ce jeune homme propulsé auprès des Grands, hésitant entre le rôle de messager et d'entremetteur. Les dernières lignes du conte prouvent s'il en était besoin que ce jeune arriviste a le sens du placement financier : 
Après avoir fait pendant quelque temps le métier de courrier, et y avoir amassé beaucoup de bien, il revint chez son père, où il n'est pas possible d'imaginer la joie qu'on eut de le revoir. Il mit toute sa famille à son aise. Il acheta des offices de nouvelle création pour son père et pour ses frères; et par là il les établit tous, et fit parfaitement bien sa cour en même temps. (Perrault, 1997)

7 En achetant des offices à ses frères et à son père, le Petit Poucet incarne donc les aspirations d'un conteur qui ne vivait pas seulement dans le monde des fées mais aussi dans celui des agioteurs, à une époque où l'argent devient roi et où la bourgeoisie montante peut acheter des titres de noblesse et redorer les blasons. Si l'ogre ne meurt pas mais se contente de perdre ses bottes, c'est sans doute qu'il ressemble à s'y méprendre à cette noblesse ruinée dont la Révolution entérinera la décadence. Bienvenue donc à ces jeunes gens qui ne se contentent plus de semer les miettes du festin sur le sentier mais comptent bien y participer sans attendre qu'un seigneur mauvais payeur vienne leur faire la grâce de rembourser ses dettes.

\section{Une première réécriture au féminin}

On oublie souvent cependant que dès le XVII ${ }^{\text {ème }}$ siècle, le personnage fut aussi décliné au féminin. Madame d'Aulnoy, contemporaine de Perrault, reine de la féerie et qui fut à l'origine de cette mode littéraire des contes de fées, publie à son tour en 1697, dans Les Contes des fées ${ }^{67}$, "Finette Cendron " avec des effets d'intertextualité voulus avec l'œuvre de son contemporain. Le conte est un amalgame du "Petit Poucet» et de "Cendrillon", salade de contes avant l'heure! Or, comme le dit si bien Philippe Hourcade, «cela commence comme le Petit Poucet mais côté fille » (D’Aulnoy, 1997 : p. 363). Ce Petit Poucet en jupons est à la fois semblable et différent de son auguste frère en littérature.

9 La trame narrative est cependant comparable même si l'écriture de Madame d'Aulnoy est plus complexe, chacun des contes ressemblant plutôt à un roman d'aventures en miniature bien éloigné de la concision d'un Charles Perrault. Notons tout d'abord un certain aristocratisme car il n'est plus question dans cette réécriture d'abandon d'enfants par des gens du peuple victimes de la famine. L'héroïne est cette fois de noble extraction car elle est fille de roi. Le conte débute en effet par les aventures de deux souverains «qui avaient mal fait leurs affaires » (D’Aulnoy, 1997 : p. 363), preuve que les nobles peuvent être sans le sou et que le prestige de la monarchie de droit divin est déjà écorné sous la plume subversive de la conteuse. Ces souverains ruinés sont donc chassés de leur royaume et condamnés pour vivre à vendre leurs nippes et leurs meubles. Définitivement ruinés et incapables de travailler, reconnaissant eux-mêmes qu'ils ne savent que «le métier de roi, qui est fort doux» (D'Aulnoy, 1997: p. 364), ils décident de perdre leurs trois filles qualifiées de "franches paresseuses qui croient être encore de grandes dames " (D'Aulnoy, $1997:$ p. 364). Notons déjà que c'est la reine qui porte la culotte et que le roi qualifié de «bon père » doit s'incliner «car la reine était la maîtresse » (D’Aulnoy, 1997 : p. 364).

10 Les rôles sont donc déjà inversés par rapport au texte de Charles Perrault dans lequel le bûcheron a certes le cœur serré à l'idée de perdre ses enfants mais s'y résigne et prend l'initiative tandis que la bûcheronne ne peut y consentir. La princesse Finette «qui était la plus petite des filles " (D'Aulnoy, 1997 : p. 364), sans que pour autant on évoque cette fois le thème du pouçot, écoute par le trou de la serrure, fâcheuse habitude qui entache 
le personnage d'un certain voyeurisme. Découvrant le noir dessein de ses parents, elle va chercher de l'aide auprès de la fée Merluche, sa marraine. Elle hérite ainsi d'un auxiliaire magique, en l'occurrence une pelote de fil inusable qu'il suffit de dévider pour marquer le chemin du retour. Nouvelle Ariane dans le labyrinthe, Finette sauve aussi ses deux sœurs bien que celles-ci la maltraitent. Le Poucet en jupons est donc sœur de Cendrillon dont elle garde la générosité, ce qui n'est pas le cas de son homologue au masculin. L'épisode se répète et la fée donne cette fois un sac de cendres magiques pour marquer la route mais en interdisant à Finette d'aider ses sœurs. Toujours généreuse, celle-ci n'obéit pas à la fée et ne peut donc plus espérer aucune aide quand l'épisode se répète une troisième fois. L'une des sœurs suggère alors d'utiliser des pois chiches le long du chemin, lesquels subiront le même sort que les miettes de pain. Le nom des deux chipies est déjà révélateur d'une certaine forme de libertinage à peine voilé. L'une s'appelle Fleur d'Amour et l'autre, Belle de Nuit, noms qui conviennent plus à des péripatéticiennes qu'à des jeunes princesses. Ce caractère libertin se retrouve chez l'héroïne elle-même. Perdue dans les bois, elle survit de manière écologique et fait pousser un gland qu'elle arrose tous les matins et tous les soirs en lui disant «crois, crois, beau gland» (D'Aulnoy, 1997: p. 371) avant de l'enfourcher enfin, et de s'y tenir longtemps, en tout cas plus longtemps que ses sœurs, ce qui est la preuve d'un certain tempérament, en le sentant ployer sous elle. Ainsi pourvue d'une autre forme d'adjuvant, Finette la rouée aperçoit enfin un jour une superbe demeure. Celle-ci se révélera être celle d'un ogre et d'une ogresse mais dans ce nouveau couple infernal, c'est une fois de plus l'élément féminin qui domine. Finette prend l'initiative de la situation. Elle pousse l'ogre dans le four où elle doit cuire le pain, obtient les bonnes grâces de la veuve plutôt joyeuse mais la décapite en la peignant. Finette est à l'image de la baronne d'Aulnoy, elle-même franche libertine. La petite rouée n'a rien à envier à ses sœurs sur certains plans et exprime des fantasmes sexuels sous une forme à peine déguisée. C'est donc au public des mondains lettrés que s'adressent ses aventures, même si la suite du texte reprend la trame de "Cendrillon " car les deux sœurs pourtant comblées de biens dans le palais des ogres s'ennuient à mourir et partent en quête de l'amour. Finette reléguée à la maison finira par retrouver le prince charmant et par l'épouser après avoir perdu une mule étrangement semblable à la pantoufle de verre.

\section{Le glissement en littérature de jeunesse via les images d'Épinal}

11 Cette version eut autant de succès à l'époque que celle de Perrault et elle fut plusieurs fois réimprimée dans la littérature de colportage, en particulier dans l'imagerie d'Épinal, ce qui fut aussi le cas de l'œuvre de Perrault. Les images d'Épinal, sorte d'ancêtre de la bande dessinée, constituées d'une série de vignettes imagées avec une légende vont contribuer largement au succès du Petit Poucet mais sous des formes réécrites, résumées et simplifiées, compte tenu du changement de support et de lectorat. Le lectorat est constitué à l'origine, au XVII ${ }^{\text {ème }}$ siècle de lettrés mondains ; celui des images d'Épinal est constitué d'adultes, lecteurs au premier niveau, voire d'illettrés qui se contentent de la belle lecture orale du colporteur et suivent avec avidité la suite des images. Annie Renonciat ${ }^{68}$ a rassemblé un corpus de 86 feuilles illustrées entre la fin du XVIII ${ }^{\text {ème }}$ et le début du XIX ${ }^{\text {ème }}$ siècle. "Le Petit Poucet » y 
trouve une place privilégiée car c'est un conte redevenu populaire par son cadre, son intrigue et les aventures du héros.

L'évolution du statut de l'enfant et le développement de l'école créent ensuite un nouveau marché pour cette littérature de colportage qui donnera lieu à récupération dans les sphères naissantes de la littérature de jeunesse. Le Petit Poucet, par l'intermédiaire des images d'épinal, va donc devenir le héros privilégié de ce nouveau public. Entre 1860 et 1870, les réécritures se succèdent, inondant ainsi l'Europe et même le monde, des aventures de ce Pouçot pour le moins doué mais stéréotypé, revu et corrigé en fonction des besoins d'un nouveau lectorat. Une fois entré dans la littérature de jeunesse, le héros semble se figer et les mêmes motifs inventés se retrouvent dès lors d'un récit à l'autre: ogre traversant les montagnes à grandes enjambées, conversation des parents autour de l'âtre à l'image du frontispice de l'édition originale des contes de Perrault, fuite de Poucet au-dessus du mur, tableaux que l'on retrouve encore dans les versions illustrées actuelles. Force est de constater que "le conte du Petit Poucet est devenu un produit stéréotypé, bâti sur ce qu'on appelle un standard qui réplique jusqu'à satiété les motifs de jadis devenus depuis belle lurette des clichés ennuyeux » (Renonciat, 1990 : p. 316). Quant au personnage lui-même, devenu un héros de littérature de jeunesse, il correspond à un modèle éducatif. Comme l'explique encore Annie Renonciat, en ce XIX ${ }^{\text {ème }}$ siècle :

la société où triomphe la concurrence érige le Petit Poucet en exemple pour sa jeunesse: héros des temps modernes, figure du courage mais aussi de la débrouillardise, voire de la roublardise, il sème dans les foyers populaires ce modèle d'intelligence, d'initiative et d'ambition, cet esprit d'entreprise qui est source d'enrichissement de la famille et de la société: message dynamique pour l'enfant-lecteur, c'est aussi un message pragmatique qui l'autorise et l'encourage à dépasser sa condition en le délivrant, si nécessaire, des interdits psychiques qui l'inhibent. Il revient à l'enfant comme au peuple de gagner sa place au soleil. Au conte d'avertissement s'oppose ce conte du dépassement où l'enfant mâle est le moteur d'une nouvelle dynamique familiale, sociale ou industrielle. (Renonciat, $1990:$ p. 317)

\section{Le petit poucet et la littérature pédagogique}

13 Il n'est donc pas étonnant que ce Petit Poucet devenu un modèle pour l'instruction des enfants du peuple ait été récupéré par les hussards de la république. Si le Petit Poucet devient à la fin du XIX ${ }^{\text {ème }}$ siècle héros de spectacle musical, voire objet de réécritures douteuses qui transforment le bûcheron en sage investisseur qui achète des terres et emploie ses enfants à les cultiver, Maurice Bouchor va le transformer en héros digne de l'école gratuite, laïque et obligatoire. À nouveau il faut se référer à l'article d'Annie Renonciat "Les Contes transcrits d'après la tradition française par Maurice Bouchor " ${ }^{69}$. Sous la troisième république les contes font une entrée officielle dans la littérature pédagogique, via les célèbres Instructions officielles en 1882 et en 1887. Le poète Maurice Bouchor va ainsi métamorphoser notre héros pour en faire un petit saint laïque susceptible de servir de modèle à des générations d'écoliers à travers un recueil adapté à toutes les circonstances, à la fois livre de classe et livre de distribution des prix. À l'aube du XXème siècle, le Petit Poucet devient ainsi un personnage digne de faire rêver les enfants mais à condition qu'on les instruise en même temps. C'est ainsi qu'est choisie la plus morale des deux fins proposées par l'auteur : 
Que notre Petit Poucet chipe à l'ogre ses bottes de sept lieues, à merveille! Mais je n'aime point qu'il se présente ensuite chez la femme de l'ogre en se disant envoyé par lui, avec le dessein de voler tout ce qu'il y a dans la maison... ${ }^{70}$

14 Aux considérations éducatives, le poète ajoute des restrictions d'ordre psychologique. Selon lui, le conte de Perrault contiendrait des épisodes fâcheux, voire trop spirituels pour les enfants. Il s'agit donc d'éviter toute scène choquante et la mort des petites ogresses est ainsi occultée car considérée comme étant d'une excessive sauvagerie. Enfin le poète ajoute de nombreux commentaires et des notes ainsi qu'une conclusion pour éviter au jeune lecteur de se fourvoyer. Annie Renonciat souligne à juste titre que le commentaire sur le Petit Poucet est exemplaire. Une introduction insiste sur les qualités du héros : son intelligence, son courage mais aussi son dévouement :

Maintenant, remarquez une chose. Avant le jour où le bûcheron et la bûcheronne perdirent leurs enfants dans les bois, le Petit Poucet peu bavard passait pour un imbécile et on lui faisait toutes sortes de misères. C'est lui, pourtant, qui fit le bonheur de la famille. Il convient de ne pas juger trop vite et surtout, il faut être toujours bon les uns pour les autres, entre frères et sœurs. (Renonciat, $1998:$ p. 94)

On serait tenté de répondre Amen à ce sermon laïque dont les présupposés sont en fait directement issus de la morale chrétienne revue et corrigée dans le creuset de la république qui a besoin de petits saints pour instruire les enfants du peuple.

\section{L'univers des multimédias}

Le Petit Poucet s'était donc déjà métamorphosé dans l'imagerie d'Épinal. Il subit cette fois une autre transformation à travers la réécriture pour la jeunesse. Or actuellement, ce sont ces mêmes versions épurées qui envahissent les rayons des librairies, voire ceux des supermarchés. Mais il faut y ajouter encore quelques traits dus non seulement à l'évolution de notre société et au statut de l'enfant mais aussi à l'apparition de nouveaux supports audio-visuels. Le XX ${ }^{\text {ème }}$ siècle voit en effet une double explosion, celle de la littérature de jeunesse et celle des nouvelles technologies : Le Petit Poucet va évoluer au gré de ces mutations. Il appartient presque exclusivement à un lectorat prioritairement enfantin, à moins qu'il ne glisse dans le monde de la publicité pour favoriser la vente de cirages ou de chaussures ou de voitures. Les Petits Poucets se multiplient donc au point que, même en suivant les petits cailloux, on risque de se perdre dans le labyrinthe des réécritures nouvelles qui visent un créneau commercial au demeurant fort rentable. Livres-objets, livres-cassettes et bien entendu récits filmiques peuplent ainsi la forêt inextricable des contes. Nous n'avons suivi que deux sentiers dans cette forêt magique, escamotant au passage les livres-disques de Bertrand Blier et de Marlène Jobert : le premier correspond aux adaptations filmiques de Michel Boisrond et d'olivier Dahan; le second nous amènera à partager les aventures de l'Enfant-océan. Chacune de ces pistes permet de prendre en compte l'évolution du personnage à travers les réécritures donnant lieu à transcodage et à néocontage depuis les seventies jusqu'à l'époque actuelle.

17 La réécriture filmique de Michel Boisrond ${ }^{71}$ date de 1972. Roger Carrel est le narrateur et c'est d'une voix enjouée qu'il évoque les aventures et les mésaventures du Petit Poucet tandis que Jean-Pierre Marielle incarne un ogre que l'on berne facilement et que le héros fait tourner en bourrique au moment de l'épisode de la poursuite. Le rire se prolonge avec une séquence ajoutée concernant le père du héros qui, affamé, tente vainement d'assommer un hérisson à l'aide d'une hache avec une telle maladresse 
qu'on en oublie le caractère épouvantable de la famine. Le même personnage se retrouve enseveli sous la neige quand il essaie de rattraper les quelques pistoles qui lui reviennent et qui sont enfouies sous la glace.

L'intrusion de la BD avec des bulles et des onomatopées est aussi censée détendre l'atmosphère et éviter au jeune spectateur d'être traumatisé par l'abandon des enfants et la rencontre avec l'ogre.

Or la réécriture filmique de Dahan ${ }^{72}$ qui a envahi le territoire depuis l'année 2001, pourtant destinée aux enfants ne joue pas du tout dans le même registre. Tout se passe comme si Dahan n'avait retenu de ce conte que l'aspect angoissant. La mise en scène privilégie le rouge vif et le noir et l'univers du récit filmique est très sombre, voire violent, à l'image de ces couleurs qui évoquent le sang et la mort. La fuite des enfants s'effectue dans une atmosphère de tempête et de bruit insoutenables. Cris et gémissements contribuent sur le plan sonore à suggérer la peur tandis que l'ogre se présente comme un monstre d'acier aux dents acérées et que les loups affamés poursuivent les enfants. Un être monstrueux en la personne d'un soldat à la jambe de fer est aussi présent, sorte de créature symbolique de la violence de la guerre, assez proche au demeurant des créatures de Madmax. Un seul qualificatif convient à ce film pourtant destiné aux enfants : terrifiant !

Pourtant le scénario demeure fidèle au conte littéraire du XVII ${ }^{\text {ème }}$ siècle. On retrouve le schéma narratif du conte et même les traits inventés par Perrault tels que l'échange des bonnets et des couronnes d'or en lieu et place d'autres coiffures, voire les célèbres bottes de sept lieues. Mais le héros se présente sous une forme dédoublée associant masculin et féminin. L'introduction du personnage de Rose permet en effet de mettre en scène un double féminisé du Petit Poucet, en quelque sorte une nouvelle Finette Cendron revue à l'aube du XXI ${ }^{\text {ème }}$ siècle. Les deux personnages ont des destins parallèles. Tous deux sont dévalorisés dans leur cercle familial parce qu'ils sont différents des autres.

21 On retrouve donc à la fois le thème du Pouçot et celui du souffre-douleur. Rose refuse de manger de la chair fraîche, ce qui en fait une ogresse elle aussi rejetée par son milieu familial. Or Rose est introduite dans le scénario dès le début. Chahuté par ses cinq frères, martyrisés par son père, le Petit Poucet est envoyé dans la sombre forêt pour ramasser du bois. Il est perdu et effrayé et c'est alors qu'il rencontre une petite fille toute de bleu vêtue qui s'appelle Rose et qui vient à son aide. Elle le rassure et lui confie un pendentif, une plume d'ange qui est un talisman et le ramène chez lui.

C'est donc elle qui devient le personnage héroïque tandis que notre héros connaît la peur. Dès lors les deux personnages vont vivre de manière conjointe toutes les aventures. Après leur première rencontre, Poucet veut retrouver Rose dans la forêt. Il la cherche en vain et c'est ainsi qu'il surprend la conversation de ses parents alors qu'il rentre dans l'humble masure. Il s'ensuit le célèbre épisode des cailloux blancs. La famille est victime de la guerre et des pillards menés par l'homme à la jambe de fer. Ces derniers ne laissent aux pauvres paysans qu'un sac de blé que les soldats du seigneur réquisitionnent au nom de la reine. Notons au passage la féminisation du pouvoir. L'intervention d'un comptable de la reine venu indemniser les victimes de guerre amène le second abandon des enfants. Poucet surpris par sa mère alors qu'il veut préventivement ramasser des cailloux se rabat sur un morceau de pain sec qui connaîtra le sort habituel des miettes. Les enfants perdus font alors face à toutes sortes de dangers et se retrouvent en plein champ de bataille. Une fois réfugié chez l'ogre, 
Poucet ne reste pas éveillé mais c'est Rose qui le réveille. C'est elle encore qui lui indique comment échapper à son père en lui retirant ses bottes de sept lieues. Elle est donc bien le pendant de Poucet mais décliné au féminin. L'insertion de cette figure féminine transforme le conte en une quête d'amour qui n'a plus grand-chose à voir avec le conte de Perrault, si ce n'est le canevas narratif. Ce n'est plus le désir d'ascension sociale qui conduit le Petit Poucet mais l'amour. À la fin, on assiste au mariage royal de Poucet et de Rose.

De plus, on se souvient que dans le conte de Perrault, le héros est bien loin d'être un modèle de vertu. Dans le film de Dahan, il devient un petit saint. Il ne joue plus de la peur de ses frères car il est lui aussi terrorisé et c'est plutôt Rose qui porte la culotte et incarne le courage et la ruse. Perrault nous proposait deux fins plus immorales l'une que l'autre. Dahan retient le dernier dénouement mais l'édulcore et exclut tout manquement à la loi. Poucet, après avoir enfilé les bottes de l'ogre arrive sur un champ de bataille. Il y découvre un capitaine agonisant qui lui remet pour la reine une lettre qui peut mettre fin au conflit. Sans ouvrir le courrier qui ne lui est pas adressé en garçon bien élevé, il transmet le message et la reine le promeut messager en lui accordant un vœu. Poucet demande simplement à revoir sa famille. Il n'est donc pas question d'argent détourné. Le dénouement du film se veut parfaitement moral. Les incarnations du mal périssent sans que le héros se salisse les mains. Le soldat pillard meurt à la guerre et l'ogre ne périt pas de la main de Poucet : un éclair s'abat sur une roche, provoque un éboulement et le fait tomber dans un ravin.

C'est donc la justice immanente qui provoque la mort des méchants tandis que les bons sont récompensés. Enfin le Petit Poucet devient le narrateur de cette histoire fort édifiante car la voix off qui ouvre et referme le récit est celle de notre héros devenu adulte, lequel, arrivé comme il le dit «au crépuscule d'une vie bien remplie » raconte sa propre histoire et commente sous forme de métalepses certains événements. Le Petit Poucet est donc devenu un vieillard sage qui a mené une vie irréprochable et qui incarne le droit à la différence et les vertus de la paix. Cette dernière réécriture est donc à l'image de nos sociétés et de ces héros de littérature de jeunesse " politiquement corrects».

\section{La réécriture romanesque contemporaine}

L'Enfant-océan de Jean-Claude Mourlevat est cependant d'une autre race! Publié chez Pocket-jeunesse, ce roman s'adresse au jeune lecteur « à partir de dix ans ", mais il peut aussi amuser le lecteur adulte et se lire à plusieurs niveaux. Cette dernière réécriture littéraire se présente comme un petit roman d'aventures divisé en chapitres, lesquels permettent de jouer avec les points de vue narratifs et de présenter sous divers angles le fil des événements. Cette réécriture est d'abord une réactualisation: Yann, le héros et ses six frères aînés, tous jumeaux, appartiennent au quart-monde et la famille Doutreleau fait l'objet de toutes les attentions des services sociaux contemporains. C'est pour fuir la violence des parents incarnée par cette horrible menace du père ( tuez les tous ») que Yann qui est bien le plus petit mais aussi le leader du groupe entraîne ses six frères dans une équipée sauvage vers l'océan, but à la fois réel et symbolique de cette quête effrénée et ponctuée d'aventures pour le moins réalistes.

Le thème de la faim déjà présent dans l'hypotexte de Perrault devient ici celui de la survie, dans une société qui n'est guère tendre avec les faibles. Certes la filiation avec 
l'hypotexte est pleinement assumée et les effets d'intertextualité sont nombreux, clins d'œil voulus qui supposent que le lecteur soit initié. La première partie s'ouvre sur cette référence en paratexte: «Le plus jeune était fort délicat et ne disait mot » (Le Petit Poucet, Charles Perrault).

Les sept garçons habitent avec leurs parents une ferme délabrée signalée par un petit panneau «Chez Perrault ».Le schéma narratif est préservé et l'on peut percevoir les motifs essentiels : misère, fuite éperdue dans un milieu hostile, rencontre d'adjuvants divers et affrontement de la force et de la cruauté d'un monstre. Mais si les thèmes originels se retrouvent encore, à savoir celui de l'abandon, celui de la faim et de la peur, celui de la préférence maternelle, celui de la victoire de l'intelligence rusée sur la cruauté, force est cependant de reconnaître que le merveilleux est volontairement escamoté au profit d'une écriture qui se veut réaliste. L'ancrage sociologique dépasse le cadre d'une simple réactualisation; le conte merveilleux laisse place au fait divers et à l'enquête policière sur fond de marginalité. L'assistante sociale, le routier «sympa » ou le boulanger charitable, sans compter les gendarmes font office d'adjuvants, tandis que le téléphone remplace les bottes de sept lieues pour ces enfants dont l'un est doté de mauvaises chaussures de femme et l'autre est un va-nu-pieds! Autre signe de la métamorphose du conte en fait divers, c'est un industriel cinquantenaire cynique qui tient le rôle de l'ogre, proclamant :

Il en va ainsi dans notre pays. Il est plus honorable de voler son prochain et de manger du hérisson comme ces gens-là que de gagner honnêtement sa vie. C'est ainsi. Mais cela changera peut-être plus tôt qu'on ne le pense. En tout cas nous y travaillons. Et nous sommes nombreux. (Mourlevat, 1999 : p. 131)

On en vient devant ce discours dont le champ lexical rejoint celui de l'extrême droite française à regretter l'ogre de Perrault sans doute moins dangereux et plus facile à berner! En ce sens le texte est profondément subversif; construit comme un véritable puzzle au fil du jeu des focalisations, il interpelle le lecteur adulte tout autant que l'enfant sur le vécu précaire de certains membres de nos sociétés contemporaines.

\section{Sur l'autre rive de la méditerranée}

En nous engageant dans la forêt des contes et en poursuivant ce Petit Poucet assez protéiforme, quelques petits cailloux ont pu nous aider au repérage. Mais notre parcours implique que l'on passe d'une rive à l'autre de la méditerranée à la poursuite de ce Poucet insaisissable, guidés cette fois par les indices de l'interculturalité ou plutôt de la transculturalité.

Deux fils d'Ariane permettent de trouver l'issue du labyrinthe sans périr dévoré sous les griffes de l'Ogre ou accablé par le nombre de ces Pouçots qui semblent être doués pour se reproduire à l'infini et parcourir de leurs bottes magiques l'univers entier. Suivons donc la piste de Mékidèche et de Baidro!

Mékidèche est à l'image de ces décepteurs, petits faibles mais rusés issus de la tradition orale algérienne, mais il entre en littérature par la grande porte, en l'occurrence celle que lui ouvre Bencheneb dans Les Contes d'Alger, recueil publié en 1944 et qui reçut le prix littéraire. Mékidèche "n'était pas plus grand qu'un nain mais il était vif et malin»! (Bencheneb, 1946 : pp. 83-91). En proie lui aussi à la jalousie, voire à la maltraitance de sa fratrie, il affronte dans son château aux murailles de cuivre rouge, la ghoule, une ogresse redoutable dont il vient à bout par la ruse à trois reprises. Il se procure ainsi 
une poule d'or et ses poussins d'argent, un tapis magnifique sur lequel s'endort l'ogresse puis parvient à la faire entrer dans une caisse en bois, sorte de cercueil auquel il met le feu, non sans avoir au passage fait en sorte que la ghoule le débarrasse de son père, de ses frères et des coépouses, laissant le champ libre au héros et à sa mère. Peut-on en l'occurrence parler d'effets d'interculturalité, compte tenu de la double culture de Bencheneb? Lui-même revendique dans son avant-propos le caractère populaire et authentiquement algérois de ces trésors mais ce serait oublier que le conte relève d'un univers transculturel et que le conte type des enfants abandonnés est présent dans le catalogue international Aarne Thompson dans la rubrique T.327 et T. 328. Quant au personnage du décepteur, on le retrouve d'un continent à l'autre !

Proche dans l'espace de Mékidèche, on trouve en effet Baidro issu quant à lui des hauts plateaux de Tiaret et transmis de génération en génération de nomades faisant paître leurs troupeaux en zone désertique! Baidro entre aussi en littérature sous la plume de Nora Aceval! Celle-ci est née en 1953 en Algérie, plus précisément à Tousnina dans la préfecture (wilaya) de Tiaret. Elle a émigré en France vers les années 1970. Elle est détentrice d'un grand patrimoine oral, hérité de son enfance passée en Algérie. Mais inlassable, elle poursuit sans arrêt, même de nos jours, son travail de collecte sur le terrain, plus particulièrement en Algérie dans la région de Tousnina, dans ce territoire rural, terre d'élection des transhumances sahariennes du grand Sud de l'Atlas saharien à l'Atlas tellien. Elle collecte, traduit, raconte les récits qui lui viennent de la tradition et qui lui ont été transmis oralement. Son œuvre est reconnue et déjà conséquente, mêlant recueils et albums. Son recueil le plus connu est intitulé Contes du Djebel Amour (2006 : pp. 130-142).

C'est au sein de ce recueil que l'on trouve un autre pouçot! L'histoire en est étrangement semblable à celle des autres! Baïdro est le dernier d'une fratrie de sept frères et malgré sa petite taille, il est le plus intelligent! Alors que ces sept garçons se retrouvent perdus, ils arrivent dans la demeure d'une famille d'ogres composée de sept filles et de leurs parents. Ayant sucé le sein de l'ogresse, le héros mérite sa protection pour toute la fratrie! Seul il accepte d'affronter l'ogre et échange la nuit venue les chéchias de ses frères contre les foulards des petites ogresses.

Comme dans le conte de Perrault, l'échange des coiffures permet aux garçons d'échapper à la mort et de s'enfuir. Mais le récit se poursuit de manière plus complexe selon un système de spirale narrative. Les six garçons, dans leur périple, rencontrent les sept filles d'un sultan grâce à Baïdro qui vient à bout de toutes les épreuves impossibles telles que boire le contenu de sept outres et l'eau de la source d'un seul coup !Au fil des épreuves imposées par le père, le héros se retrouve face à l'ogre dont il doit voler successivement la bague, le tapis de prière, et la jument magique. Baïdro parviendra à rendre l'ogre aveugle et à le faire entrer dans un coffre de bois! Il épouse alors la princesse préférée du sultan et devient son successeur, illustrant une fois de plus la fonction narrative du mariage royal !

Peut-on une fois de plus se poser le problème de l'interculturalité car Nora Aceval, notre contemporaine est franco-algérienne et revendique une double culture ? Certes elle recueille sur les lèvres des dernières conteuses des contrées désertiques les trésors de l'oralité en voie de disparition mais c'est pour les transcrire ou plutôt les écrire sous une forme littéraire et leur redonner une nouvelle vie grâce au néocontage qu'elle pratique auprès de cercles divers qui vont de la diaspora maghrébine en France au public lettré! Mais elle est aussi le fruit d'une culture littéraire française, titulaire 
d'une maîtrise de lettres au sein de l'université française où elle a fait ses études! Comment retrouver dans ce cas les petits cailloux alors que la transculturalité inhérente au monde des contes s'amuse à brouiller les pistes?

Les réécritures du petit poucet ont donc servi de premier fil d'Ariane dans cette quête ou plutôt cette enquête vouée à rester incomplète; certes l'hypotexte de Charles Perrault semble bien prégnant et l'intertextualité est encore visible à notre époque en particulier dans le secteur florissant de la littérature de jeunesse. L'évolution des supports du conte constitue une deuxième clé de cette enquête bien incomplète. Que ce soit à travers les images d'Épinal ou les versions filmiques, le héros se métamorphose inévitablement en passant non plus de l'oralité à l'écrit mais de la forme écrite à des supports imagés! Mais il se transforme surtout au gré des mutations sociologiques et s'adapte à un lectorat sans cesse nouveau au fil de l'évolution de nos sociétés.

Héros des temps modernes, tantôt petit saint, tantôt arriviste sans scrupules, le Petit Poucet est à notre image et peut donc encore vivre de nombreuses aventures. Intergénérationnel, il est aussi transculturel et se métamorphose en enfant-doigt au Niger sous la plume de Boubou Hama (1984: pp. 72-78) ou en Mékidèche ou en Baidro de l'autre côté de la Méditerranée! Ainsi vivent et revivent les contes !

\section{BIBLIOGRAPHIE}

ACEVAL Nora, Contes du Djebel Amour. (Recueil), Paris, Ed Seuil, 2006.

BENCHENEB Saadeddine, Contes d'Alger, Oran, Henrys, 1946.

D'Aulnoy Marie-Catherine, Contes, Les Contes de fées; Contes II, Contes nouveaux ou Les Fées à la mode, édition du tricentenaire, introduction par Jacques Barchilon, texte établi et annoté par Philippe Hourcade, Paris, Société des Textes Français Modernes, diffusion Klincksieck, 1997-98.

GENETTE Gérard, Palimpsestes, Paris, Seuil, 1982.

MOURLEVAT Jean-Claude, L'Enfant-océan, Paris, Pocket-jeunesse, 1999.

RENONCIAT Annie, «Les Contes transcrits d'après la tradition française par Maurice Bouchor », in Actes du colloque du tricentenaire de Charles Perrault, Les Grands Contes du XVII ${ }^{\text {ème }}$ siècle et leur fortune littéraire, sous la direction de Jean Perrot, Paris, In Press Editions, 1998, (p.85-96). -«Petit Poucet dans la jonchée des feuilles », Bulletin de la société archéologique, historique et artistique Le Vieux Papier, Paris, fascicules 316 et 317, avril et juillet 1990.

BOUBOU Hama et MARIKo Keletegui, « contes du monde entier », in Contes du Niger, Paris, éditions Nathan Afrique, 1984, pp. 72-78. 


\section{NOTES}

67. Madame d'Aulnoy, édition du tricentenaire, introduction par Jacques Barchilon, texte établi et annoté par Philippe Hourcade, Paris, Société des Textes Français Modernes, diffusion Klincksieck, Contes I, Les Contes de fées, 1997,604 p. ; Contes II, Contes nouveaux ou Les Fées à la mode, 1998, 577p. Le conte de "Finette Cendron» se trouve dans le premier volume, p.363-385. Signalons aussi Madame d'Aulnoy, Contes des fées suivis des Contes nouveaux ou Les Fées à la mode, édition critique par Nadine Jasmin, « bibliothèque des Génies et des Fées », Paris, Champion, 2004, 1220 p.

68. Nous nous référons à l'étude comparative menée par Annie Renonciat, «Petit Poucet dans la jonchée des feuilles ", Bulletin de la société archéologique, historique et artistique Le Vieux Papier, Paris, fascicules 316 et 317, avril et juillet 1990.

69. Annie Renonciat, «Les Contes transcrits d'après la tradition française par Maurice Bouchor », dans Actes du colloque du tricentenaire de Charles Perrault, Les Grands Contes du XVIIème siècle et leur fortune littéraire, sous la direction de Jean Perrot, Paris, In Press Éditions, 1998, p. 85-96.

70. Citation de Maurice Bouchor, in Annie Renonciat (1998 : p. 90).

71. «Le Petit Poucet », film réalisé par Michel Boisrond, France, 1972.

72. Le Petit Poucet, film réalisé par Olivier Dahan, France, 2001. Pour retrouver le scénario et les séquences de ce film, on peut se référer à l'article de Juliette Sales, «Poucet de peur... et de poésie », dans la revue Synopsis, $\mathrm{n}^{\circ}$ 16, décembre 2001, pp. 78-80.

\section{RÉSUMÉS}

On pourrait donc rappeler dans un premier temps comment ce personnage du Petit Poucet, issu des traditions populaires subit une nouvelle métamorphose en se glissant avec subtilité dans la mode des contes de fées qui envahit les salons et les milieux mondains à la fin du XVII ${ }^{\text {ème }}$ siècle. Il nous semble aussi intéressant de montrer le glissement du personnage dans la littérature de jeunesse où il devient une sorte de petit saint laïque. La dernière partie de cette étude, à la fois synchronique et diachronique, pourrait porter sur les réécritures de ce conte dans les multimédias au XX ${ }^{\text {ème }}$ et au XXI ${ }^{\text {ème }}$ siècle. On s'intéressera ainsi au film de Michel Boisrond qui date de 1972, qu'il est intéressant de comparer à la réécriture filmique de Dahan en 2001 et à la récente réécriture de Jean-Claude Mourlevat, L'Enfant-océan. Enfin, dans une dernière partie, il ne faudrait pas aussi oublier que ce personnage est non seulement un héros intergénérationnel et intertextuel mais qu'il est aussi un héros universel et transculturel et qu'on le retrouve sous la forme de Mékidèche et de Baïdro au Maghreb.

First we might recall how Tom -Thumb, born from popular traditions had undergone a new transformation by slipping subtly into the fashion of fairy tales which invaded salons and fashionable circles at the end of the $17^{\text {th }}$ century. It seems to us interesting to show the slipping of the character into youth literature in which he becomes a sort of small holy layman. The last part of this study, both synchronic and diachronic, might deal with the rewriting of this tale in the $20^{\text {th }}$ and the $21^{\text {st }}$ multimedia. In this way we'll be interested in Michel Boisrond's film which dates back to 1972 and which is interesting to compare with Dahan's filmic rewriting and with Jeanclaude Mourlevat's rewriting, The Ocean-Child. Finally, in a last part, we should not forget that this character is not only an inter-generational and inter-textual character but a universal 
and cross-cultural hero and that we encounter him under the form of Mekideche and Baïdro in Maghreb.

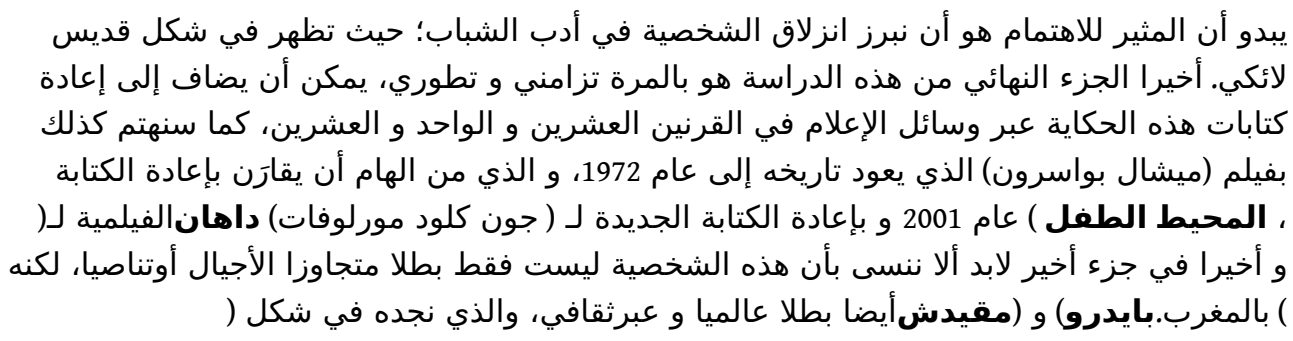

INDEX

Keywords : inter-textuality, cross-culture, rewriting, tale, Mékidèche, Baïdro

$$
\text { التناص, عبر الثقافي, إعادة الكتابة, الحكايةفهرس الكلمات المفتاحية: }
$$

Mots-clés : intertextualité, transculturalité, réécriture, conte, Mékidèche, Baïdro

\section{AUTEUR}

\section{MARIE-AGNÈS THIRARD}

Professeur émérite - Université Lille 3 - Laboratoire ALITHILA - France 